\title{
A 3D model analysis of patellofemoral joint: effect of anterior translation and internal rotation of tibia
}

\author{
Michihiko FUKUNAGA*, Kentaro MORIMOTO*, Tomoko KAJIWARA*, \\ Kuniyuki ITO* and Ryuji NAGAMINE** \\ * Department of Innovative Engineering, Oita University \\ 700 Dannoharu, Oita 780-1192, Japan \\ E-mail: fukunagam@oita-u.ac.jp \\ ** Center of Joint Arthroplasty and Rheumatism, Fukuoka Tokushukai Hospital \\ 4-5 Sugukita, Kasuga, 816-0864, Japan
}

Received: 28 April 2017; Revised: 7 June 2017; Accepted: 28 June 2017

\begin{abstract}
The objective of this study was to find out the effect of tibial position and orientation to patellofemoral kinetics, especially during deep knee flexion. The data about patellofemoral kinetics is necessary for calculating tibiofemoral joint force which is used to evaluate a design of knee prosthesis, however, there have been no data how it changes by tibial anterior translation or internal rotation which recent prostheses are aiming. We performed a three-dimensional model analysis with axioms of point contact and force equilibrium. The flexion angle was from $0^{\circ}$ to $150^{\circ}$ by four conditions. The neutral condition was based on the two-dimensional analysis using PS type of the prosthesis, without internal rotation and plenty femoral roll back. The other conditions were with over $20 \mathrm{~mm}$ of tibial anterior translation, with $15^{\circ}$ of tibial internal rotation, and both the translation and rotation during deep knee flexion. As a result, patellofemoral joint force reduced by tibial anterior translation and did not change by tibial rotation. Tensile force of patella tendon did not change by the tibial position/orientation. By rotating tibia internally, medial contact force increased and lateral reduced. Moreover, tibial anterior translation facilitated the dislocation of patellofemoral joint, and lateral side lifted off with tibial internal rotation. The contact force reduced rapidly before the lift off, and medial contact force increased by the lateral lift off. In this case, although the magnitude of the patella tendon force did not change, the direction of the force might change unignorably. Both tibial anterior translation and internal rotation might be necessary to be capable of deep knee flexion, however, it is also necessary to discuss the interaction between tibiofemoral and patellofemoral joints for evaluating the new prosthesis intending deep knee flexion.
\end{abstract}

Keywords : Biomechanics, Orthopaedics, Deep knee flexion, Patellofemoral joint, Mathematical model, Three-dimensional analysis, Kinetics

\section{Introduction}

Deep knee flexion is defined as the motion or posture with over $130^{\circ}$ of knee flexion angle with some external force. The typical motion is sitting on a floor directly, e.g.: squatting, kneeling, cross-leg sitting or sedentary sitting. Such motions are usually seen in our daily lives in some regions in Asia and Arab because their traditional lifestyles are without chair or bed. Moreover, a kneeling posture is necessary for religious praying of Islam or Buddhism. Even in other regions, deep knee flexion is necessary for high QOL (quality of life) in taking bath, putting on/off socks, resting outside or gardening. However, conventional artificial knee joint cannot flex deeply. Therefore, knee prostheses of the next generation are expected to be capable of flexing deeply.

For designing and evaluating the new type of prosthesis, it is necessary to prove the safety and effectiveness before 
applying it to patients. The problem is that there are little data of kinematics/kinetics of deep knee flexion which are necessary for evaluating a new design. There have been the reports about a knee kinematics during deep knee flexion by radiographic method (e.g.: Hefty et al., 1998 or Tamaki et al., 2008), however, there are still little data about the kinetics of deep knee flexion. Although the data of in-vivo measurement of joint load are reported recently (Kutzner et al., 2010), the data about deep knee flexion are not included.

In this study, we targeted patellofemoral joint, because the research about patellofemoral joint are even less than that of tibiofemoral. Patellofemoral kinetics might be very important, as patella is connecting quadriceps and patellar tendon which applying its tensile force to tibia. As for tibiofemoral joint, there has been some research about patellofemoral joint kinetics (e.g.: Ishimaru et al., 2014 or Sharma et al., 2017). Kinetics data would not be obtained by just observing patients, and some dynamic simulation should be performed, except the recently reported results using patella component with force sensor during an operation, called "trial" (e.g. Kawahara et al., 2012 or Konno et al., 2014), or little have reported in-vivo measurement (Sharma et al., 2008).

We have already performed a two-dimensional model analysis of a total knee including tibiofemoral and patellofemoral (Fukunaga et al., 2009), however, it was the analysis using PS type of knee prosthesis which had not be able to make deep knee flexion and performed deep knee flexion imaginary in order to find out the reason why conventional prostheses cannot make deep knee flexion. The result was that PS type of knee prosthesis cannot propel the femoral rollback during deep knee flexion (Fukunaga et al., 2011), and tibia might translate more anterior in the case of capable of deep knee flexion. Moreover, it is generally noted that tibia rotates internally by knee flexion, which is called screw home movement. Such tibial position and orientation would affect patellofemoral kinetics strongly, and the effects would return to tibiofemoral. Therefore, it is necessary to find out the effect of anterior translation and internal rotation of tibia to patellofemoral kinetics for discussing the kinetics of tibiofemoral joint during deep knee flexion.

In this study, we performed a three-dimensional model analysis of patellofemoral joint by changing the position and orientation of tibia, and calculated the patellofemoral kinematics and kinetics, especially about the forces around patella.

\section{Materials and Methods}

\subsection{Outline and conditions of the model}

Our model was based on the model of Wismans et al. (1980) and Hirokawa (1985). It included patella, femur, quadriceps and patellar tendon as shown in Fig.1. In this model, the contact surfaces were assumed to be solid, and contacting by two points on medial and lateral sides. The friction forces were neglected. The contact surfaces were modeled by parametric surfaces. The fixed coordinate axis $(O-x y z)$ was on femur and patellar coordinate axis $\left(O^{\prime}-X Y Z\right)$ was moving.

We settled two conditions in the model. First condition was that the contact surfaces contacted at medial and lateral points. The condition of a point contact could be written as Eq.(1)-(2). Here $\mathbf{c}$ stands for the position vector of a contact point, $\mathbf{a}$ for the position vector of patellar origin, $\mathbf{T}$ for the rotation matrix from patellar axis to femoral axis, and $\boldsymbol{\delta}$ for

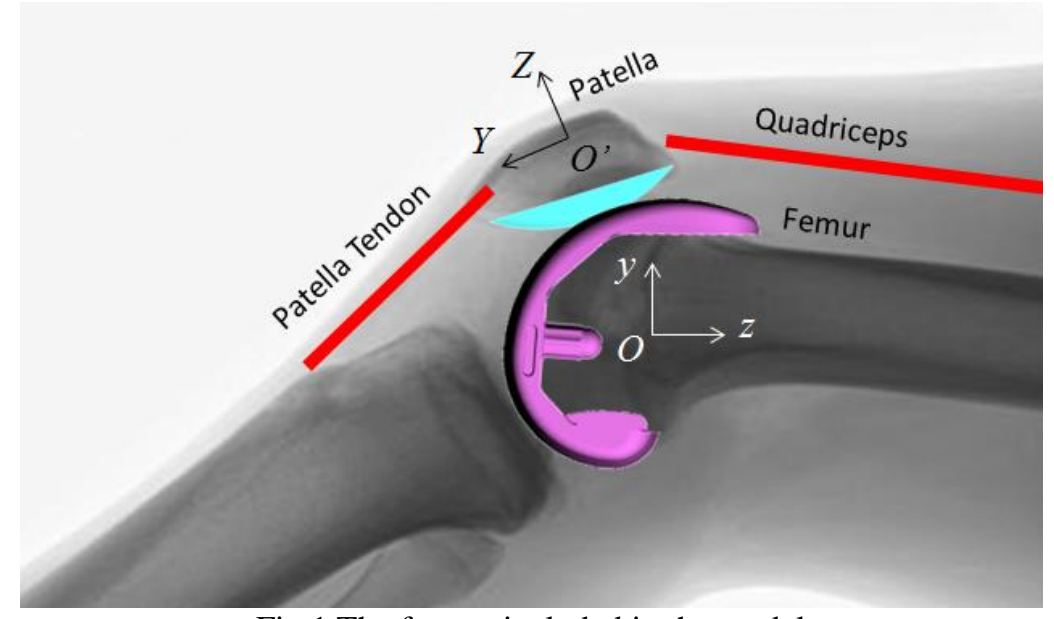

Fig. 1 The factors included in the model 
the position vector of a contact point on patellar coordinate. And $\mathbf{n}$ stands for the normal vector of femoral surface on a contact point and $\boldsymbol{\tau}_{r}$ and $\boldsymbol{\tau}_{s}$ for the orthogonal tangential vectors of patellar surface on a contact point. Equation (1) represented that the surfaces were not dislocated, and Eq.(2) that the surfaces were not overlapped.

$$
\begin{aligned}
& \mathbf{c}=\mathbf{a}+\mathbf{T} \boldsymbol{\delta} \\
& \mathbf{n} \cdot \mathbf{T} \boldsymbol{\tau}_{r}=0 \quad \mathbf{n} \cdot \mathbf{T} \boldsymbol{\tau}_{s}=0
\end{aligned}
$$

Second condition was the force and moment equilibrium. These could be represented as Eq.(3)-(4), and shown in Fig.2. Here $f$ and $F$ stand for the tensile forces of ligaments and muscle forces, $m$ and $M$ for the number of the fibers of ligaments and muscles, $\mathbf{v}$ and $\mathbf{v}_{m}$ for the directional vectors of ligaments and muscles and $\boldsymbol{\rho}$ and $\boldsymbol{\rho}_{m}$ for the position vectors of patellar insertions of the ligaments and muscles respectively. The direction vectors could be represented as Eq.(5), where $\mathbf{r}$ stands for the position vector of femoral insertion of soft tissues. Muscle forces were inputted, and the tensile forces were calculated from the length of ligaments. Ligaments were modeled as nonlinear springs as shown in Eq.(6), as inputting constant of spring $k$ and natural length $l_{0}$. And $M_{r} \lambda$ was the moment around medial-lateral axis. Because this model was the reconstruction of tibiofemoral model (Fukunaga et al., 2008) and the tibiofemoral model was designed as to input the knee flexion angle, such moment had been left. In this study, the moment was converged zero by adjusting the inputting angle around $X$ axis.

$$
\begin{aligned}
& \sum_{i=1}^{2} p_{i} \mathbf{n}_{i}+\sum_{i=1}^{m} f_{i} \mathbf{v}_{i}+\sum_{i=1}^{M} F_{i} \mathbf{v}_{m i}=\mathbf{0} \\
& \sum_{i=1}^{2} p_{i}\left(\mathbf{T} \boldsymbol{\delta}_{i}\right) \times \mathbf{n}_{i}+\sum_{i=1}^{m} f_{i}\left(\mathbf{T} \boldsymbol{\rho}_{i}\right) \times \mathbf{v}_{i}+\sum_{i=1}^{M} F_{i}\left(\mathbf{T} \boldsymbol{\rho}_{m i}\right) \times \mathbf{v}_{m i}+M_{r} \boldsymbol{\lambda}=\mathbf{0}
\end{aligned}
$$

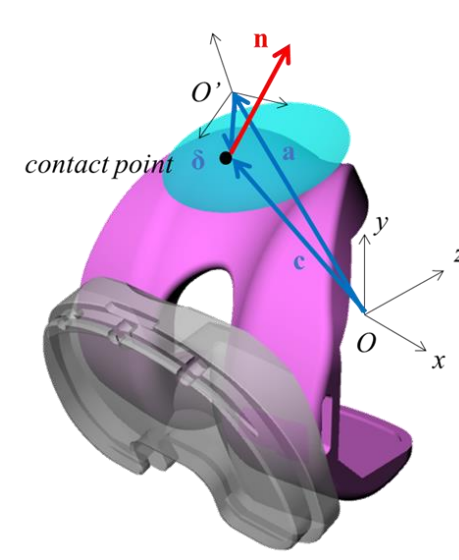

Contact force

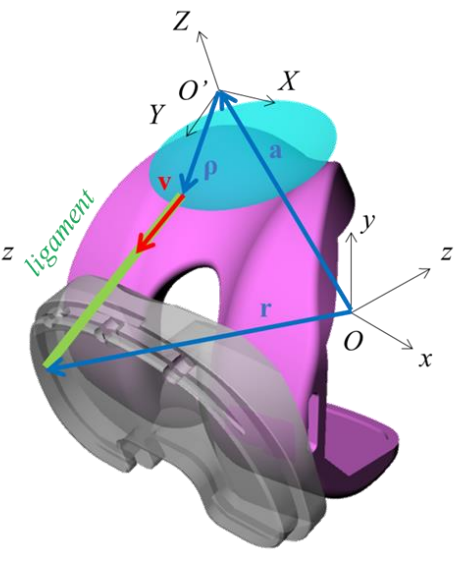

Tensile force of ligaments

Fig. 2 Forces acting on patella

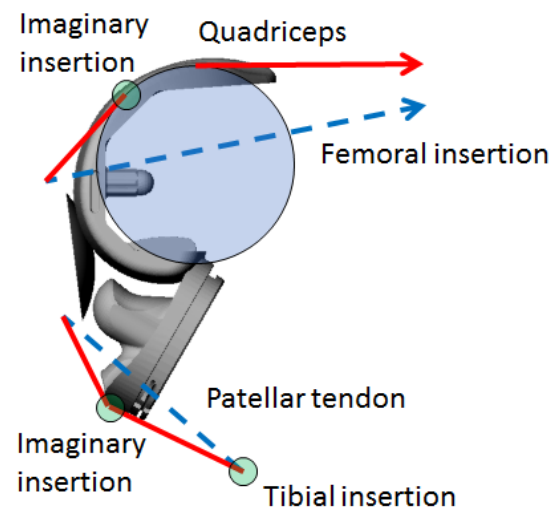

Fig.3 Soft tissues were bent by tibial and femoral components 


$$
\begin{aligned}
& \mathbf{v}=\frac{(\mathbf{r}-\mathbf{a}-\mathbf{T} \boldsymbol{\rho})}{|\mathbf{r}-\mathbf{a}-\mathbf{T} \boldsymbol{\rho}|} \\
& f_{i}=\left\{\begin{array}{cc}
k_{i}\left(l-l_{0}\right) & \left(l>l_{0}\right) \\
0 & \left(l \leq l_{0}\right)
\end{array}\right.
\end{aligned}
$$

The total number of equations were 16 when contacting at two points. The simultaneous equation was solved by Newton-Raphson method, and the unknown quantities were outputted; position/orientation of patella, position of contact points on femur and patellar and contact forces.

When a knee flex, quadriceps contact with patellar groove and is bent. The same phenomenon would occur for patellar tendon and the anterior corner of a tibia. Thus, such bending was represented by using imaginary position of insertion, as shown in Fig.3. Patellar groove was modeled as a cylinder to set the femoral imaginary insertion of quadriceps on the tangent, and the tibial imaginary insertion of patellar tendon was set on the anterior corner of tibia.

\subsection{Inputting data for the analysis}

The contact surface of femoral component was constructed from Scorpio Super Flex PS-type, Stryker Co. for a right leg. Patellar surface was modeled as circular dome shape, structed by referring the catalog of Stryker Co. The geometries are shown in Fig.4. The contact surfaces were modeled as the parametric surfaces dividing the femoral side because the surface is large and could not modeled as a single surface formula. Soft tissues included in this model was patellar tendon and quadriceps. These were modeled as the bundles of 8 and 13 fibers respectively, as shown in Fig.5. Patellar tendon was dealt with as a ligament, since it connects patella and tibia.

The simulation was performed from $0^{\circ}$ to $150^{\circ}$ of flexion angle. Magnitude of quadriceps force was inputted as a unit force, because there were no external forces in the model and the contact forces and the tensile force of patella tendon could be obtained as the ratios to quadriceps force. The positions of insertion of tibial side of patellar tendon were determined by the results of previous reports of the PS type of knee prosthesis using a two-dimensional model

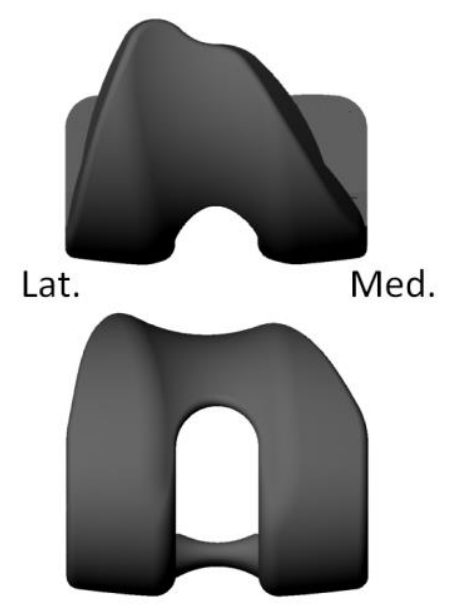

Femoral component

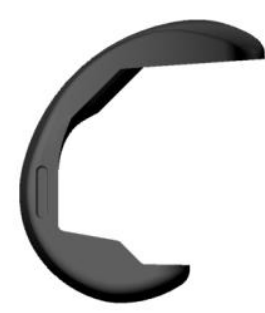

Patellar component

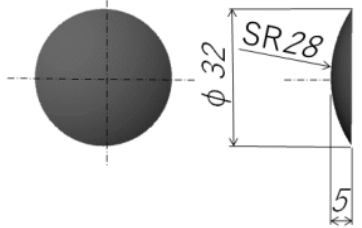

Fig.4 The knee prosthesis used for the simulation

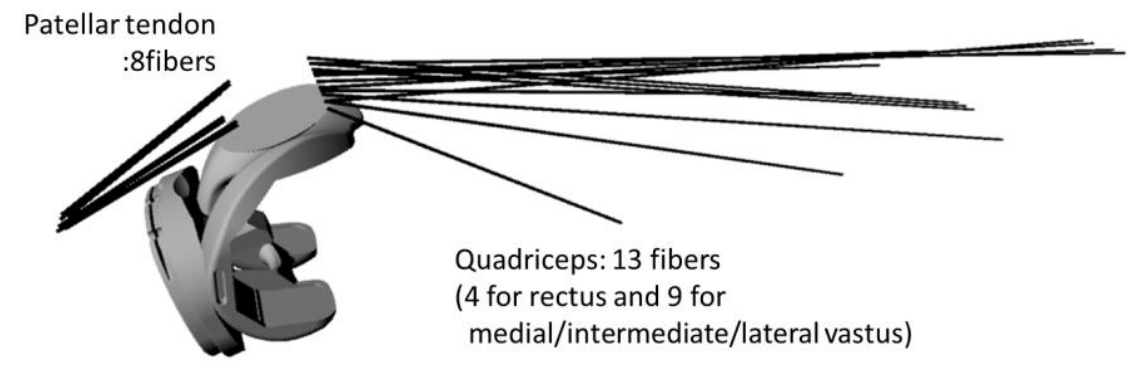

Fig.5 Modeled patellar tendon and quadriceps 
analysis (Fukunaga et al., 2009). Since the objective of this study was to evaluate the effect of tibiofemoral motion, the positions were modified by translating or rotating tibia. First, tibia was translated anterior, because the femoral roll-back of a PS type of knee prosthesis might be not enough in deep flexion. Tibia translated gradually, as the distance was $20 \mathrm{~mm}$ at $130^{\circ}$ of flexion angle, which the translation quantity was determined by the report that the tibiofemoral joint of a normal knee might be in subluxation in deep knee flexion. The direction was vertical to the tibial axis on a sagittal plane. Second, tibia was rotated internally, because the ordinal data were just two-dimensional and a normal knee rotates internally by flexing the knee, which is called screw home movement. The rotation angle was $15^{\circ}$ and the rotation axis crossed the contact point on the post with cam. Thus, four conditions were settled as lined up below. In case of both rotation and translation, tibia rotated after anterior translation. Inputted positions of the tibial insertions of patellar tendon are shown in Fig.6.

(1) Neutral: two-dimensional flexion

(2) Translating tibia anterior

(3) Rotating tibia internally without anterior translation

(4) Rotating tibia internally with anterior translation
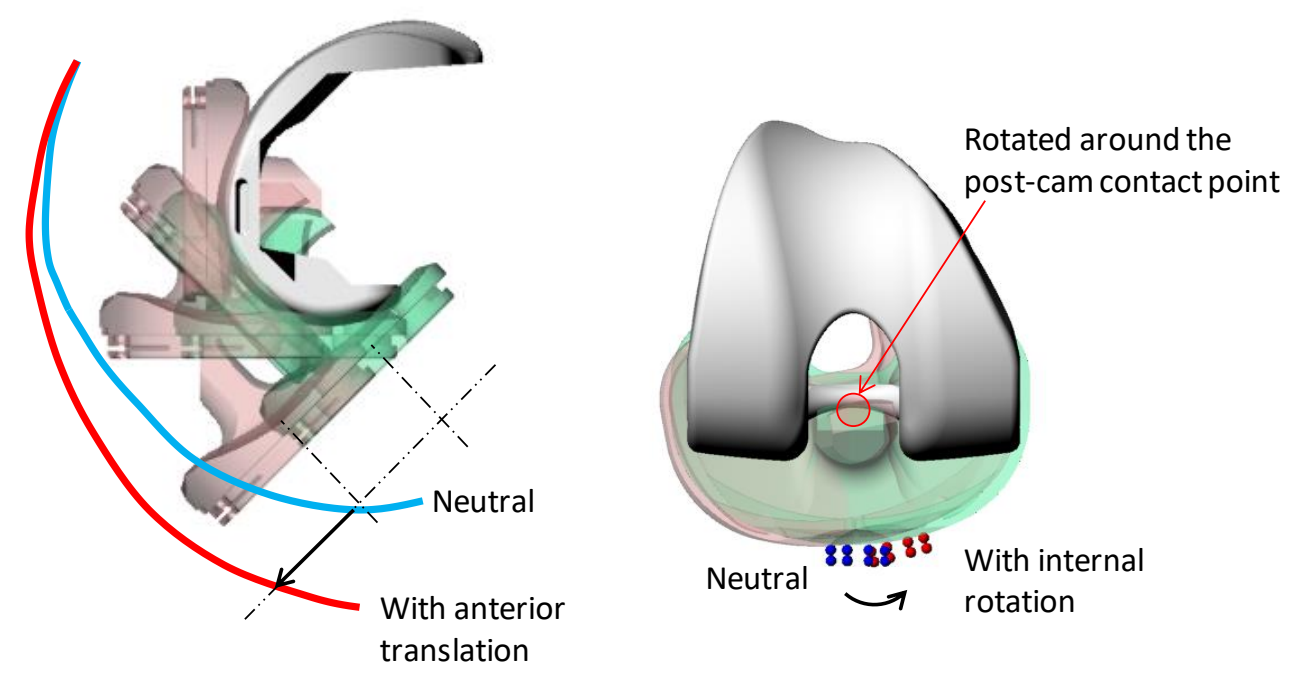

Fig.6 Input position of the tibial insertion of patellar tendon

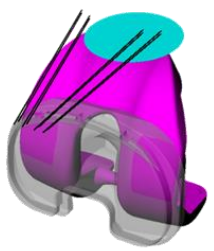

$0^{\circ}$

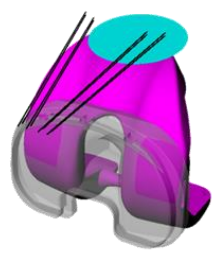

$0^{\circ}$

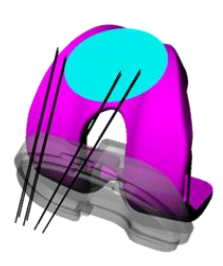

$30^{\circ}$

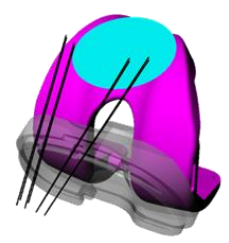

$30^{\circ}$

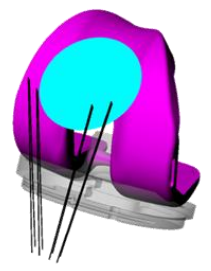

$60^{\circ}$

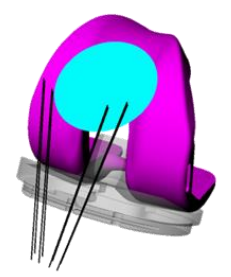

$60^{\circ}$

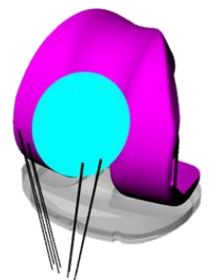

$90^{\circ}$

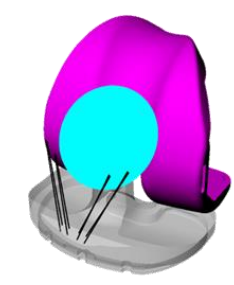

$90^{\circ}$

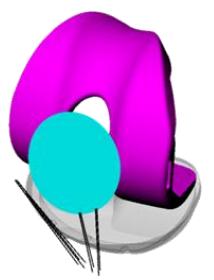

$120^{\circ}$

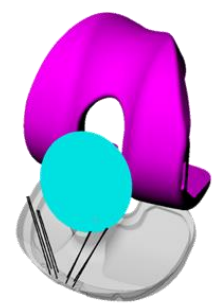

$120^{\circ}$

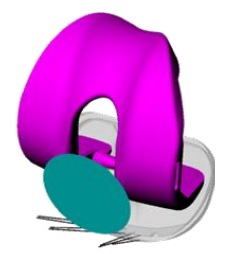

$150^{\circ}$

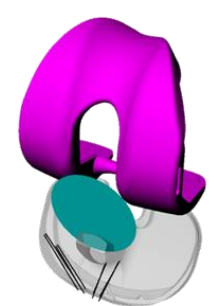

$150^{\circ}$

With anterior translation

Fig.7 Position/orientation with and without tibial anterior translation 


\section{Results}

As a result, the calculations converged to their results for all the conditions except over $142^{\circ}$ of flexion angle with both tibial anterior translation and internal rotation. The position/orientation of the components are shown in Fig.7 and Fig.8, which shows the effect of tibial anterior translation and internal rotation respectively. With tibial translation and rotation, patellofemoral articular surfaces dislocated. Our model could not reproduce such lift off, and the surfaces

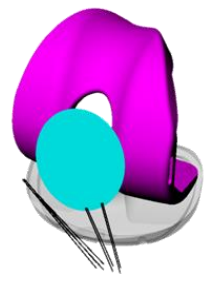

$120^{\circ}$

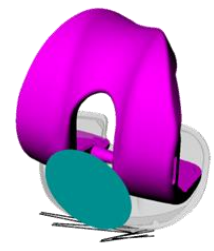

$150^{\circ}$

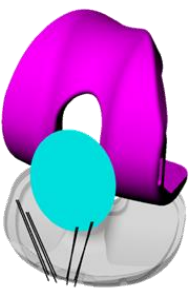

$120^{\circ}$

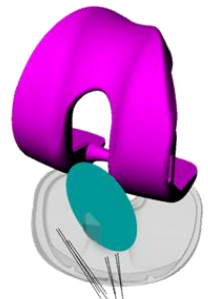

$142^{\circ}$

Without anterior translation

With anterior translation

Fig.8 Position/orientation with $15^{\circ}$ of tibial internal rotation

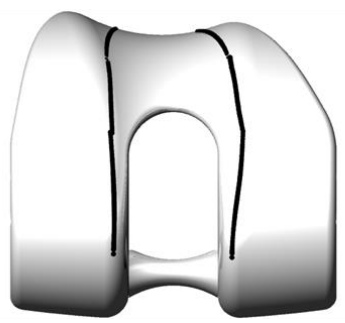

$\leftarrow$ Lat.

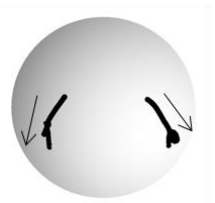

Neutral

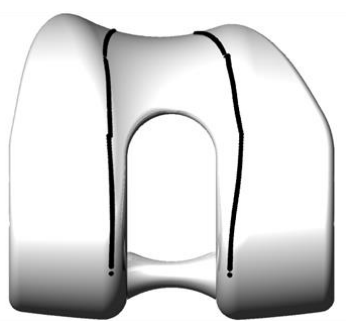

Femoral side

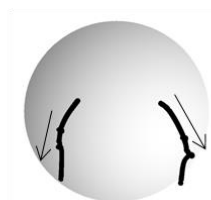

Pan
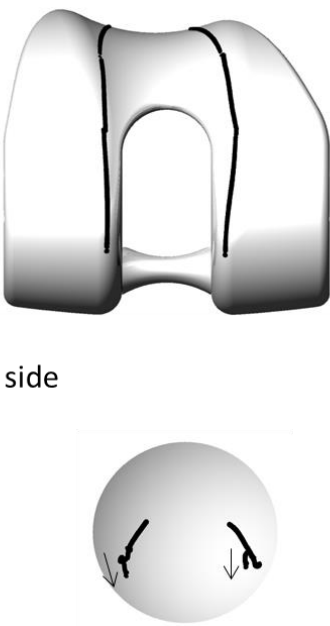

Internal rotation

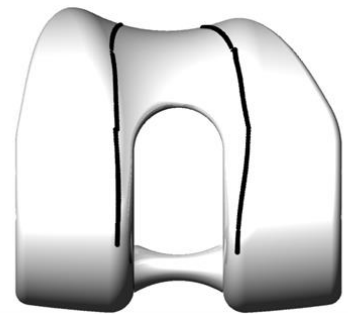

Med.-

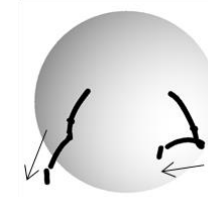

Translation\&rotation

Fig.9 Contact point trajectories

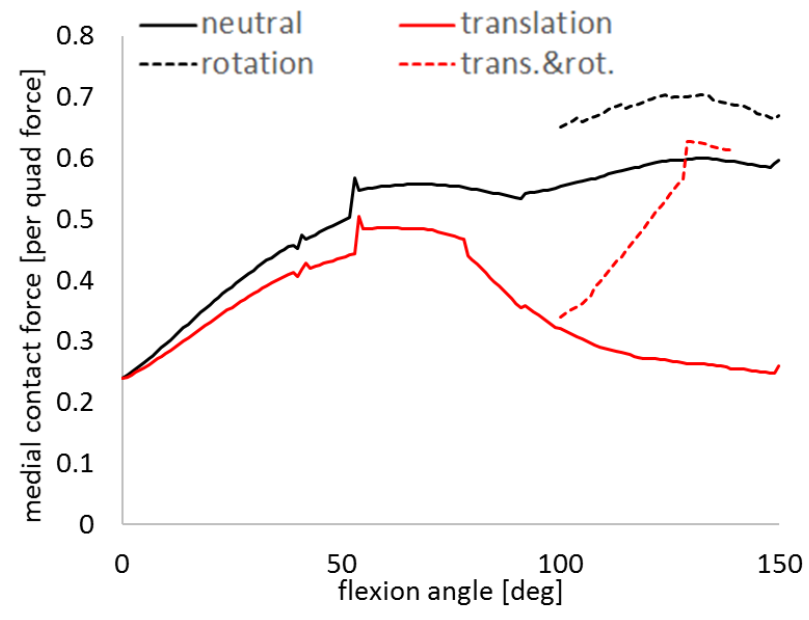

Medial

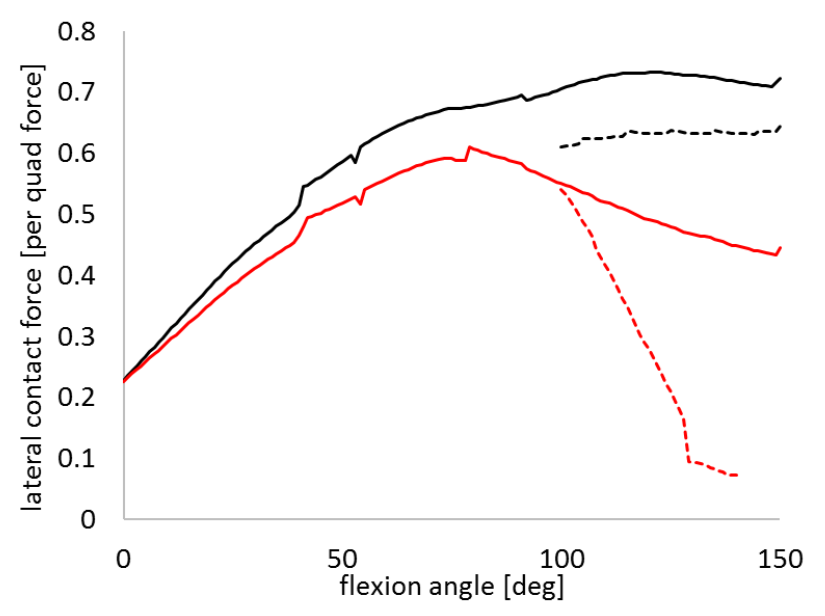

Lateral

Fig.10 Contact forces on medial/lateral contact points 
contacted on out of the supposed surface where also modeled as the polynomial surface. It might be the cause for the divergence of calculations. And it could also be recognized that the post on tibia overlapped with patellar component, though our model did not include the contact of post and patella.

The trajectories of contact points are shown in Fig.9. Contact points on patellar side were on the edge of the contact surface during deep flexion with tibial anterior translation. Moreover, lateral contact point was completely out of the surface by anterior translation and internal rotation. There were no notable differences on femoral side.

Medial and lateral contact forces are shown in Fig.10. Medial force was smaller than lateral in neutral case, and the medial was larger than lateral when tibia rotated internally. With both anterior translation and internal rotation, lateral contact force reduced rapidly and medial force increased, which might be caused by the lift off on lateral contact surfaces, and medial contact force to be subjected the patellofemoral force on one point.Patellofemoral joint forces and the tensile forces of patellar tendon were shown in Fig.11. By the tibial anterior translation, patellofemoral joint force reduced and a tensile force of patellar tendon did not change much. These were not affected by tibial rotation.

\section{Discussions}

First, the results were compared to the previous studies to examine the validity of our model. Figure 12 shows our results by blue lines and the results of previous research which were summarized by Mason et al. (2008) of patellofemoral joint forces and patellar tendon forces relative to the quadriceps force. Since there were no data for deep flexion, the data of Sharma et al. (2008) were added by black lines which show normal knee and two types of prostheses. All the data including ours were roughly in good agreement with each other, though our calculated joint force was larger than the others without tibial anterior translation.
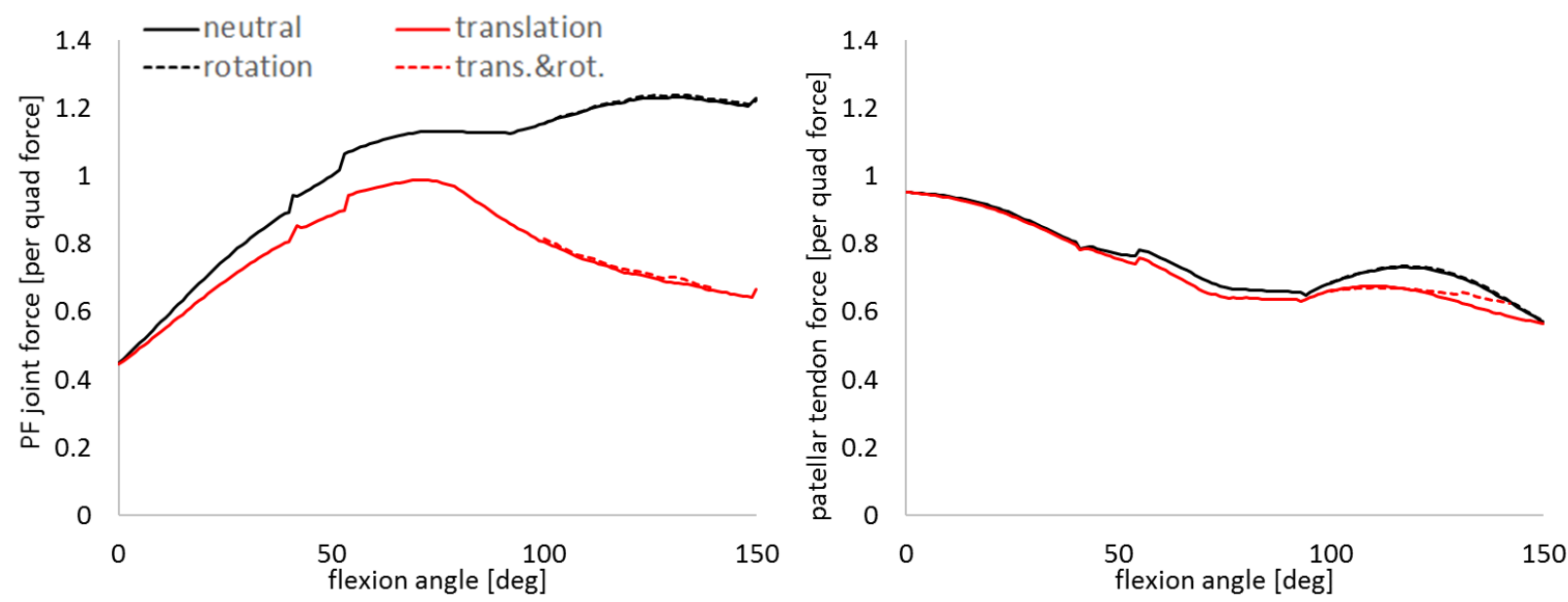

Fig.11 Patellofemoral joint forces and patellar tendon forces
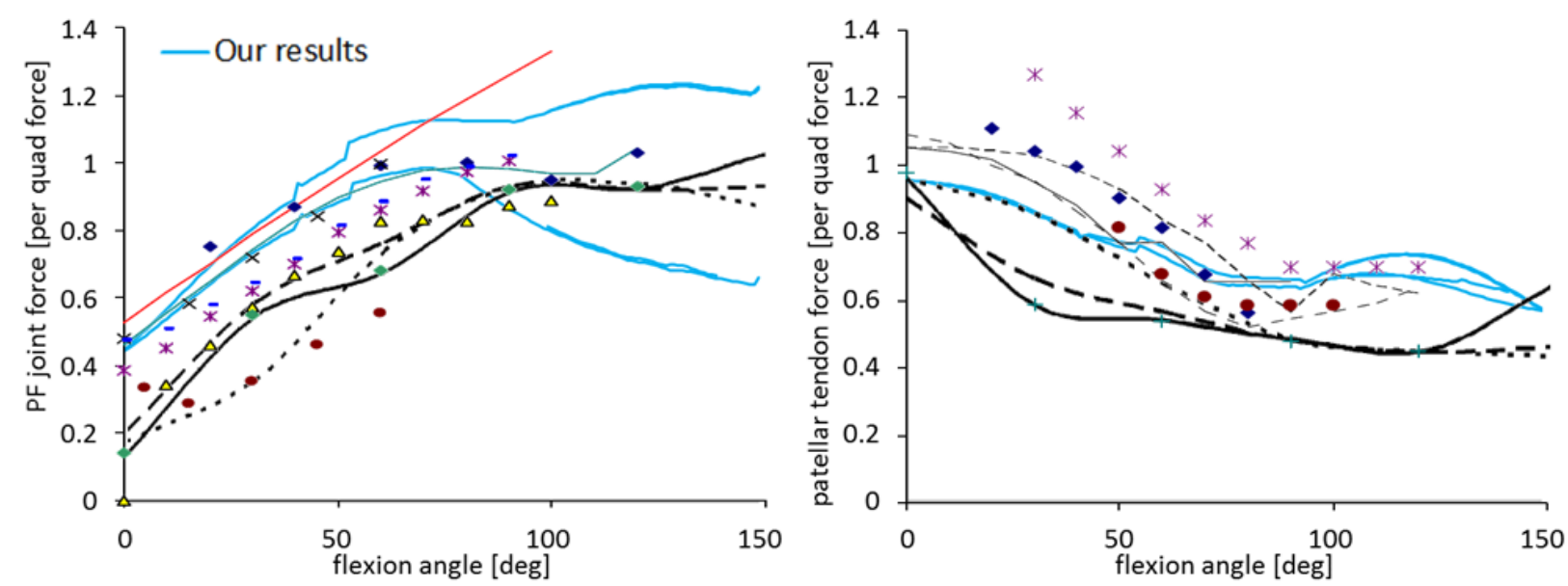

Fig. 12 Comparing our results with previously reported data

(This figure includes the figure of Mason et al., 2008 and Sharma et al., 2008) 
By translating tibia anterior, patellofemoral joint force reduced and the tensile force of patella tendon did not change. This can be explained using the force equilibrium of three forces in sagittal plane (Fukunaga et al., 2009). There were three forces acting on patella, quadriceps force, patella tendon force and contact force. When three forces are in moment equilibrium, these forces might cross on a single point, and the ratio between the forces could be determined by the angles among the forces by force equilibrium condition. By translating tibia anterior, the angle between quadriceps and patella tendon expanded and the angles between contact force and the other two forces contracted. The force ratio between quadriceps and contact force was determined by the ratio between the cosines of the contracting two forces, thus the ratio between quadriceps and patella tendon did not change. Since the force acting on tibia is patella tendon force, it could be considered that the tibiofemoral force would not change by tibial translation or rotation. However, since the moment arm length or the direction of the force changed by the tibial motion, the tibiofemoral force or contact would change. Therefore, patellofemoral kinetics still could not be ignored if the ratio between quadriceps and patella tendon forces would not change.

It should be noted that the contact forces on medial and lateral contact points varied by tibial rotation. The ratio of lateral contact force to medial is shown in Fig.13. It shows that lateral contact force was larger than medial in neutral condition, however, these forces came nearer by rotating tibia $15^{\circ}$. As mentioned above, tibia usually rotates internally by flexing knee, thus it could be considered that the medial and lateral contact balance of patellofemoral joint came better by the physiological motion of tibia, which supported Konno et al. (2014) indicated. Besides, when tibia translated anterior, the ratio of contact forces increased in high and deep flexion, with both medial and lateral forces reduced. In condition of both translation and rotation, the balance was lost by the lateral lift off, however, it could be speculated that there might be the proper quantity of translation and rotation to achieve the medial/lateral balance. The medial/lateral balance of contact forces might change by the design of the artificial knee joint, both patellar groove and patellar component. Assuming the optimized design of biological object, a normal knee would move as to achieve the medial/lateral balance, or the surfaces were designed as to move biologically. Then the best design of patellofemoral might be bio-imitating, however, tibiofemoral would not be able to design as so because of the ligaments and cartilages. Analyzing the effect of patellofemoral geometrical design is our future task.

Although it was not modeled, it should also be noted that the results indicated the contact of post on tibia and patella. They would not contact when the knee flex by the post-cam contact as its design concept, however, tibia possibly translated anterior when the knee tended flex deeply. It possibly is one of the reasons why conventional prosthesis cannot flex deeply.

It has been generally noted that knee joint cannot make deep knee flexion without plenty femoral roll back and tibial internal rotation, however, the interaction of patellofemoral and tibiofemoral joint should be considered for evaluating a new type of knee prosthesis to be capable of deep flexion, as the kinetics around a knee would change drastically from a normal knee or conventional prostheses. Moreover, the reason why conventional artificial knee joint cannot make deep knee flexion would be attributable to the interaction. Our future task is to create the analytical model including both tibiofemoral and patellofemoral joints, which can deal with deep knee flexion.

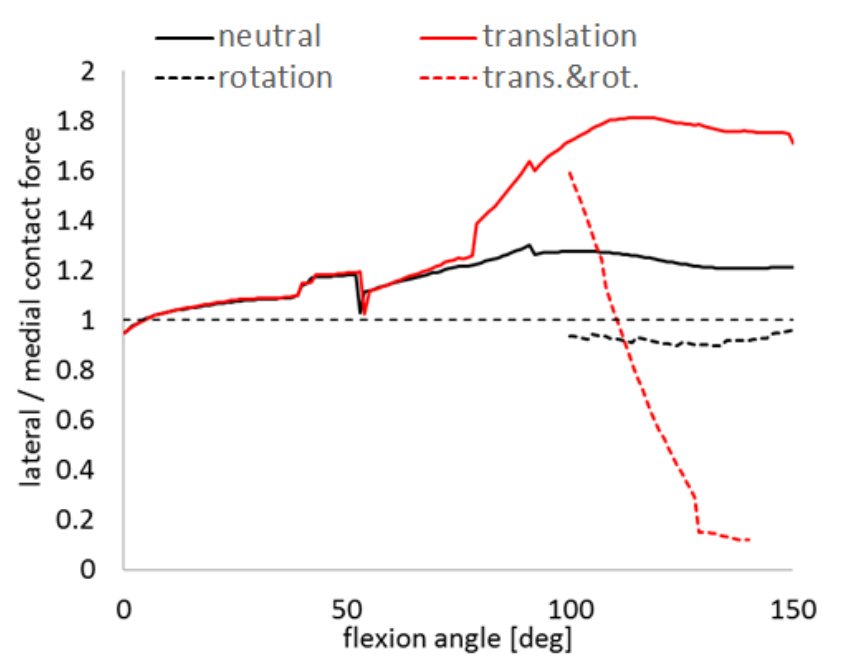

Fig.13 Ratio of lateral to medial contact force 


\section{Conclusion}

We performed a 3D model analysis of patellofemoral joint with changing the tibial position and orientation. As a result, tibial anterior translation reduced patellofemoral joint force and patella tendon force was not affected. Tibial internal rotation changed the balance of medial and lateral contact forces, and promoted the lift off of lateral contact surface when tibia translated anterior. Our future tasks are to reconstruct the model to reproduce the lift off, and to analyze deep knee flexion by the model including tibiofemoral joint.

\section{Acknowledgement}

This study was supported by JSPS KAKENHI Grant Number JP16K16463.

\section{References}

Fukunaga M., Katsuhara T., Hirokawa S., A 3D model analysis of artificial knee joint during passive deep flexion, Memoirs of the Faculty of Engineering, Kyushu University, Vol.68, No.1 (2008), pp. 139-148.

Fukunaga, M., Katsuhara, T., Hirokawa, S., Mawatari, M. and Hotokebuchi, T., A 2D model analysis of artificial knee joint during deep squatting, Journal of Biomechanical Science and Engineering, Vol.4, No.2 (2009), pp.298-305.

Fukunaga, M., Kawanoya, J., Hirokawa, S., Control of femoral rollback of PS knee prosthesis through slip ratio, Tribology Online, Vol.6, No.1 (2011), pp.32-35.

Hefzy, M. S., Kelly, B. P. and Cooke, T. D. V., Kinematics of the knee joint in deep flexion: a radiographic assessment, Medical Engineering and Physics, Vol.20, No.4 (1998), pp.302-307.

Hirokawa, S., Analytical study on three-dimensional model of femoropatellar joint, Journal of the Society of Instrument and Control Engineers, Vol.21, No.4 (1985), pp.411-417. (in Japanese)

Ishimaru, I., Shiraishi, Y., Ikebe, S., Higaki, H., Hino, K., Onishi, Y., Miura, H., Three-dimensional motion analysis of the patellar component in total knee arthroplasty by the image matching method using image correlations, Journal of Orthopaedic Research, Vol.32, No.5 (2014), pp.619-626.

Kawahara, S., Matsuda, S., Fukagawa, S., Mitsuyasu, H., Nakahara, H., Higaki, H., Shimoto, T., Iwamoto, Y., Upsizing the femoral component increases patellofemoral contact force in total knee replacement, The Journal of Bone and Joint Surgery, Vol.94-B, No.1 (2012), pp.56-61.

Konno, T., Onodera, T., Nishio, Y., Kasahara, Y., Iwasaki, N., Majima, T., Correlation between knee kinematics and patellofemoral contact pressure in total knee arthroplasty, Journal of Arthroplasty, Vol.29, No.12 (2014), pp.2305-2308.

Kutzner, I., Heinlein, B., Graichen, F., Bender, A., Rohlmann, A., Halder, A, Beier, A., Bergmann, G., Loading of the knee joint during activities of daily living measured in vivo in five subjects, Journal of Biomechanics, Vol.43, No.11 (2010), pp.2164-2173.

Mason, J. J., Leszko, F., Johnson, T., Komistek, R. D., Patellofemoral joint forces, Journal of Biomechanics, Vol.41, No.11 (2008), pp.2337-2348.

Sharma, A., Leszko, F., Komistek, R. D., Scuderi, G. R., Cates H. E., Liu F., In vivo patellofemoral forces in high flexion total knee arthroplasty, Journal of Biomechanics, Vol.41, No.3, (2008), pp.642-648.

Sharma, A., Grieco T. F., Zingde S. M., Dennis, D. A., Anderle N. R., Komistek, R. D., In vivo three-dimensional patellar mechanics - normal knees compared with domed and anatomic patellar components, The Journal of Bone and Joint Surgery, Vol.99, No.5 (2017), pp.e18(1-13).

Tamaki, M., Tomita, T., Watanabe, T., Yamazaki, T., Yoshikawa, H. and Sugamoto, K., In vivo kinematic analysis of a high-flexion, posterior- stabilized, mobile-bearing knee prosthesis in deep knee bending motion, Journal of Arthroplasty, Vol.24, No.6 (2009), pp.972-978.

Wismans, J. A. C., Veldpaus, F., Janssen, J., Huson, A., Struben P., A three-dimensional mathematical model of the human knee joint, Journal of Biomechanics, Vol.13, No.8 (1980), pp.677-685. 